\title{
A meta-analysis comparing 48-week treatment outcomes of single and multi-tablet antiretroviral regimens for the treatment of people living with HIV
}

Patrick G. Clay ${ }^{1 *}$, Wei C. Yuet ${ }^{1}$, Christiane H. Moecklinghoff ${ }^{2}$, Inge Duchesne ${ }^{3}$, Krzysztof L. Tronczyński ${ }^{4}$, Sandip Shah ${ }^{5}$ and Dong Shao ${ }^{5}$

\begin{abstract}
Objectives: To compare outcomes with single tablet regimens (STR) versus multi-tablet regimens (MTR) for human immunodeficiency virus (HIV) treatment using published data.

Design: Systematic review and random-effects meta-analysis of literature on approved and investigational HIV regimens.
\end{abstract}

Methods: The research followed the Preferred Reporting Items for Systematic Reviews and Meta-Analyses guidelines. Single or un-blinded studies reporting a direct comparison between STR and MTR were eligible for the meta-analysis. Double-blinded studies were excluded due to lack of difference in pill burden between cohorts. The key outcomes of interest included: adherence rates/proportion meeting target, efficacy, safety/tolerability, non-clinical and economic outcomes.

Results: After screening 63 full-text articles and posters, 14 studies were eligible for the meta-analysis. The analysis showed that patients taking STR had improved outcomes over those taking MTR. Patients were significantly more adherent regardless of daily dosing frequency (odds ratio [OR]: 1.96, $\mathrm{p}<0.001$ ) and were more likely to achieve virological suppression (relative risk [RR]: 1.05, $\mathrm{p}=0.002$ ). There was a trend toward a lower discontinuation risk in the STR cohort, together with reported higher therapy satisfaction, better symptom control, improved health status, reduced healthcare resource utilization and demonstrated cost-effectiveness compared to MTR. There were no differences in CD4 cell count increase (at 48 weeks) or safety outcomes.

Conclusions: The findings of this study confirm previously reported preliminary findings of the advantages of STR over MTR for HIV treatment in adherence, therapy continuation, viral suppression, tolerability, quality of life improvement, cost-effectiveness and healthcare resource utilization.

Keywords: Human immunodeficiency virus, Treatment adherence and compliance, Treatment outcome, Quality of life, Economics pharmaceutical

\footnotetext{
*Correspondence: Patrick.Clay@unthsc.edu

1 University of North Texas System College of Pharmacy, 3500 Camp

Bowie Blvd, Fort Worth, TX 76107, USA

Full list of author information is available at the end of the article
} 


\section{Introduction}

Although treatment options have expanded significantly, human immunodeficiency virus (HIV) remains an important global public health issue. In 2017, the number of people living with HIV (PLWH) was approximately 36.9 million [1] and about 1.1 million people died of acquired immune deficiency syndrome (AIDS)-related illnesses [1]. The annual rate of new infections remained relatively constant between 2005 and 2017, however, the number of PLWH receiving antiretroviral therapy (ART) increased dramatically from 2.2 million to 21.7 million [1].

ART has evolved significantly in the past two decades. Beginning with protease inhibitors in 1995, through multiple drug single class combination tablets in 2001 and multiple drug, multiple class combination tablets in 2006, to the most recent and sixth ART class, post-attachment inhibitors (ibalizumab), in 2018. Today, multiple class, multiple drug, fixed-dose, single tablet regimens (STR) dominate use [2]. Collectively, these advances have improved outcomes, have enhanced tolerability and reduced pill burden and enabled more PLWH to reach goal adherence levels [3]. With each newly approved STR, there is potential for treatment guidelines/practice patterns to change in PLWH naive to therapy, yet the needs of the individual is paramount $[4,5]$. Efforts continue to develop long-acting mechanisms to deliver ART that may improve durability $[3,6]$.

Simplified regimens improve adherence and clinical outcomes and have long been proven in other conditions, such as hypertension, diabetes and asthma [3, 7]. With the advent of STR, HIV has become a treatable, chronic disease [8] with both the Joint United Nations Programme on HIV and AIDS (UNAIDS) and the World Health Organization (WHO) highly recommending once daily fixed-dose combinations (FDC) to improve adherence $[6,9]$.

FDC can include multiple classes of ART and may represent an entire recommended regimen taken once daily (e.g., one 'tablet' once daily). FDC reduce the number of total daily pills (pill burden) and contain at least two active agents, rather than a single dosing unit with a single active agent with a pharmacokinetic enhancer/ booster, such as Kaletra ${ }^{\mathrm{TM}}$ (Iopinavir/ritonavir), Prezcobix $^{\text {TM }}$ (darunavir/cobicistat [DRV/COBI]) and Evotaz ${ }^{\text {TM }}$ (atazanavir/cobicistat [ATV/COBI]) [10].

When a FDC constitutes an entire regimen and is provided as one 'tablet' for use once daily, it is known as an STR. However, FDC can be co-administered with another ART agent to create a multiple-tablet regimen (MTR). MTR can be taken once or more times per day, dependent upon the MTR components. Since 2014, a number of STR and MTR have been approved and are now recommended by treatment guidelines as first-line treatment for treatment-naïve patients. The newly included STR regimens include elvitegravir/cobicistat/emtricitabine/tenofovir alafenamide (EVG/COBI/FTC/TAF), darunavir/cobicistat/emtricitabine/tenofovir alafenamide (DRV/COBI/FTC/TAF) and emtricitabine/rilpivirine/ tenofovir alafenamide (FTC/RPV/TAF). MTR component agents include ATV/COBI, DRV/COBI and emtricitabine/tenofovir alafenamide (FTC/TAF). As none of these combinations have been compared in a systematic review [11], the objective of this study is to compare the clinical (adherence, virologic and safety/tolerability), economic and non-clinical or humanistic outcomes of all currently available STR and MTR.

\section{Methods}

\section{Search strategy and study selection}

A literature review and meta-analysis was conducted to include all currently approved FDC, both STR and MTR. The study followed the Preferred Reporting Items for Systematic Reviews and Meta-Analyses (PRISMA) guidelines and the PICOS principles (Patient, Intervention, Comparator, Outcome, and Study Design) based on an internal study protocol (available upon request) [12]. Databases including Embase, PubMed and ClinicalTrials.gov were searched to capture relevant literature published from 2005 to 2017, with filters to include only studies conducted in humans and published in English.

The endpoints of interest included adherence, efficacy, safety and healthcare resource utilization along with an emerging element for decision makers, humanistic outcomes [11]. Data included published, publicly available randomized controlled trials (RCT) and observational studies. Case studies, abstracts without posters or fulltext, letters, reviews, editorials and comments were excluded. Two reviewers conducted a two level screening process. A first-pass screening of bibliographic details, titles and abstracts of all citations eliminated duplicates and irrelevant studies. Full-text of studies meeting the eligibility criteria and reported outcomes of interest were included for data extraction and screened for inclusion. For all quantitative outcomes, only studies with outcome measures in evaluable format $(\mathrm{n} / \mathrm{N}$, mean, standard deviation, $\mathrm{N}$ or median and inter-quartile range) with a clear comparison between STR and MTR arms were included. Double-blind studies were excluded as enrolled subjects would receive the same number of tablets, thus removing direct comparison between MTR and STR. Qualitative evidence data capture reporting in the literature precluded use of this standard, thus data was included when provided and resulted in slight variance in studies used. For all studies however, the methodological quality of RCTs was assessed using the Cochrane handbook, 
focusing on the risk of bias across five different categories (selection, performance, detection, reporting and attrition) [13]. For observational studies, the Critical Appraisal Skills Programme (CASP) Cohort Study Checklist was used to evaluate the overall study quality [14]. A random-effects meta-analysis was conducted using Comprehensive Meta-Analysis Software (version 2) for data analysis and to create the forest plots.

\section{Endpoints}

The 48-week primary endpoints were used in all analyses. Adherence outcomes included achieving a protocol specific threshold measure (yes/no, dichotomous) and as author reported adherence frequency (pill count, percentage adherence or proportion of days covered, etc.). Efficacy outcomes included the percentage of patients achieving viral load suppression (i.e., $<50$ copies $/ \mathrm{ml}$ ) and changes in mean CD4 counts from baseline. Safety outcomes included the percentage of patients experiencing any severe adverse event (SAE), mortality or any grade 3 to 4 clinically significant event or laboratory abnormality. Tolerability outcomes included the percentage of patients discontinuing their STR or MTR for any reason. Economic and humanistic outcomes are summarized in the review to provide a single-source review of STR compared with MTR to inform healthcare providers as well as policymakers.

\section{Statistical methods}

Inverse variance methods were used in a random-effects model to analyze both dichotomous and continuous data and to assess heterogeneity [15]. Heterogeneity was evaluated using the Chi squared test and quantified using the $\mathrm{I}^{2}$ statistic [16]. Alpha $<0.05$ was used to determine statistical significance. $\mathrm{I}^{2}$ values of 25,50 and $75 \%$ correspond to low, medium and high levels of heterogeneity, respectively. Summary statistics were calculated for each study to describe observed treatment effects; mean and standard deviation values were calculated where studies reported median and inter-quartile range. When necessary, 95\% confidence intervals $(\mathrm{CI})$ were converted to standard deviations via the formula $\mathrm{SD}=(\sqrt{ } \mathrm{N}) *(($ upper limit - lower limit)/3.96). A pooled treatment effect estimate was then calculated as the weighted average of the treatment effects estimated in the individual studies. Each study was weighted as the inverse of the variance of the effect estimate (i.e., one over the square of its standard error). Larger studies with smaller standard errors were given more weight than smaller studies with larger standard errors. For the studies which had multiple MTR arms, data from the MTR arms were first pooled within the trials and then between the trials. Dichotomous outcomes were evaluated by making an adjustment to the study weights according to the extent of variation, or heterogeneity, among the treatment effects.

Values for dichotomous outcomes (adherence [based on a threshold measure; yes/no], viral load suppression, safety events, and tolerability) were presented as $n / N$, where $n=$ subset of sample size; $N=$ total sample size, and the odds ratio (OR) or risk ratio (RR) with 95\% CI were calculated. Values for continuous outcomes (CD4 cell counts and adherence [based on pill count or percentage of $\operatorname{drug}(\mathrm{s})$ used]) were presented as mean, standard deviation (SD) and N (sample size), with calculated standardized mean differences. For humanistic (qualitative) data, a global approach was adopted to rate the results as negative, neutral or positive based on the change in scores from baseline to the end of the study period. For economic evaluations where studies reported healthcare resource use (HRU), the direct medical costs and incremental cost-effectiveness ratio (ICER) values were summarized.

\section{Results \\ Study details}

The literature search yielded 4002 citations, of which 287 were duplicates, resulting in 3715 unique records. After screening titles and abstracts, 192 potentially relevant studies were identified. Nine additional articles were found from hand searching of bibliographies. Following a careful examination of the 201 full-text articles and clinicaltrials.gov records, a total of 63 studies (34 RCT, 24 observational studies (OS) and five economic studies) met the inclusion and exclusion criteria and were included for qualitative evidence synthesis. The PRISMA flow of the review process is shown in Fig. 1. Nearly half $(n=30)$ of the studies were conducted in treatment naïve PLWH, 22 studies included treatment experienced PLWH and 11 included both treatment experienced and naïve PLWH.

Twenty-eight of the 63 studies were not included in the previous meta-analysis [11]. Most of the studies not included in the previous review reported efficacy (73\%) and safety/tolerability (59\%). Adherence was reported in $48 \%$ of the added studies. Fewer reported (or assessed) humanistic measures (patient-reported outcomes [PRO]) (14\%) and economic endpoints (21\%).

Key characteristics of the 63 studies are summarized in Additional file 1: Table S1 Of these 63 studies, only 14 studies reported outcome measures in evaluable format $(\mathrm{n} / \mathrm{N}$, mean, standard deviation, $\mathrm{N}$, or median and inter-quartile range) and/or at consistent evaluation time points, with a clear comparison between STR and MTR, and were included for meta-analysis. Baseline demographics of the population in the 14 studies are shown in Additional file 2: Table S2. 


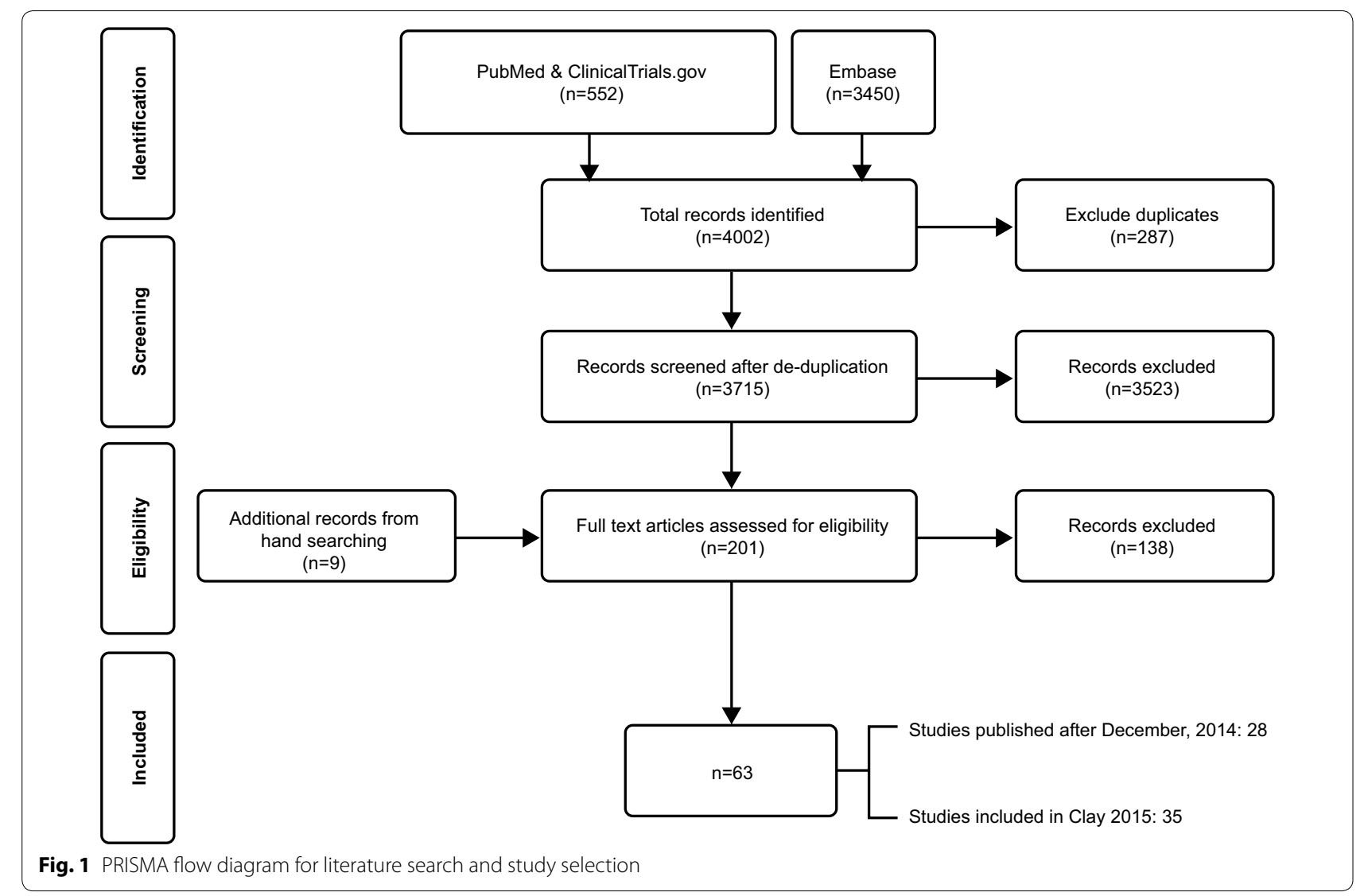

The majority of the included RCTs had low risk of bias, with potentially high risk for blinding in treatment allocations. The overall quality of all observational studies was determined as medium and satisfactory. The detailed assessments are presented in Additional file 3: Table S3.

\section{Adherence outcomes}

While 30 of the 63 studies reported patient adherence outcomes, only eight studies reported quantifiable data and were included in the meta-analysis [17-24]. Seven of these eight studies [17-23] reported patient adherence as a dichotomous outcome (threshold defined per study protocol), two reported mean difference in medication adherence calculated using pill count and one reported using both formats $[17,24]$.

In the dichotomous adherence outcome analysis, adherence was significantly better in patients receiving STR than patients receiving once or twice daily MTR: $55.4 \%$ (range: $25.7 \%$ to $91.6 \%$ ) versus $42.0 \%$ (range: $15.1 \%$ to $85.3 \%$ ), odds ratio (OR) of adherence: $1.96,95 \%$ confidence interval $(\mathrm{CI}) 1.66-2.33, \mathrm{p}<0.001$. No heterogeneity was observed $\left(\mathrm{Chi}^{2}=4.91 ; \mathrm{i}^{2}=0.0 \%\right)$ (Fig. 2a).

In the sub-analysis of STR versus once daily MTR, adherence was significantly better in patients receiving
STR compared with those receiving once daily MTR: $80.5 \%$ (range: $76.5 \%$ to $85.4 \%$ ) versus $71.8 \%$ (range: $68.0 \%$ to $75.0 \%$ ), OR: 1.66, 95\% CI 1.21-2.27, p=0.002; Fig. 2a).

In the sub-analysis of STR versus twice daily MTR, adherence was numerically better in patients receiving STR compared with those receiving twice daily MTR: $84.1 \%$ (range: $82.6 \%$ to $85.4 \%$ ) versus $66.8 \%$ of patients (range: $60.7 \%$ to $73.9 \%$ ), OR: 2.53 , 95\% CI $1.13-5.65$, $\mathrm{p}=0.02$; Fig. 2a).

Medication adherence based on "pill count" (two studies) was higher in the STR group (92.1\% [range: $86.0 \%$ to 98.3\%]) compared with $84.8 \%$ (range: $73.6 \%$ to $95.9 \%$ ) in the collective MTR groups. The standardized mean difference (SMD) comparing medication adherence was also statistically significantly in favor of the STR group (SMD: 0.68, 95\% CI 0.40-0.97, $\mathrm{p}<0.001$ ) in the two studies (Fig. 2b).

\section{Efficacy outcomes}

Twenty-four of the 63 studies reported efficacy data for viral load suppression and CD4 count. Eighteen studies were excluded from the meta-analysis since they reported time points other than 48 weeks or parameters not in a quantifiable format. Six studies [22, 25-29] 


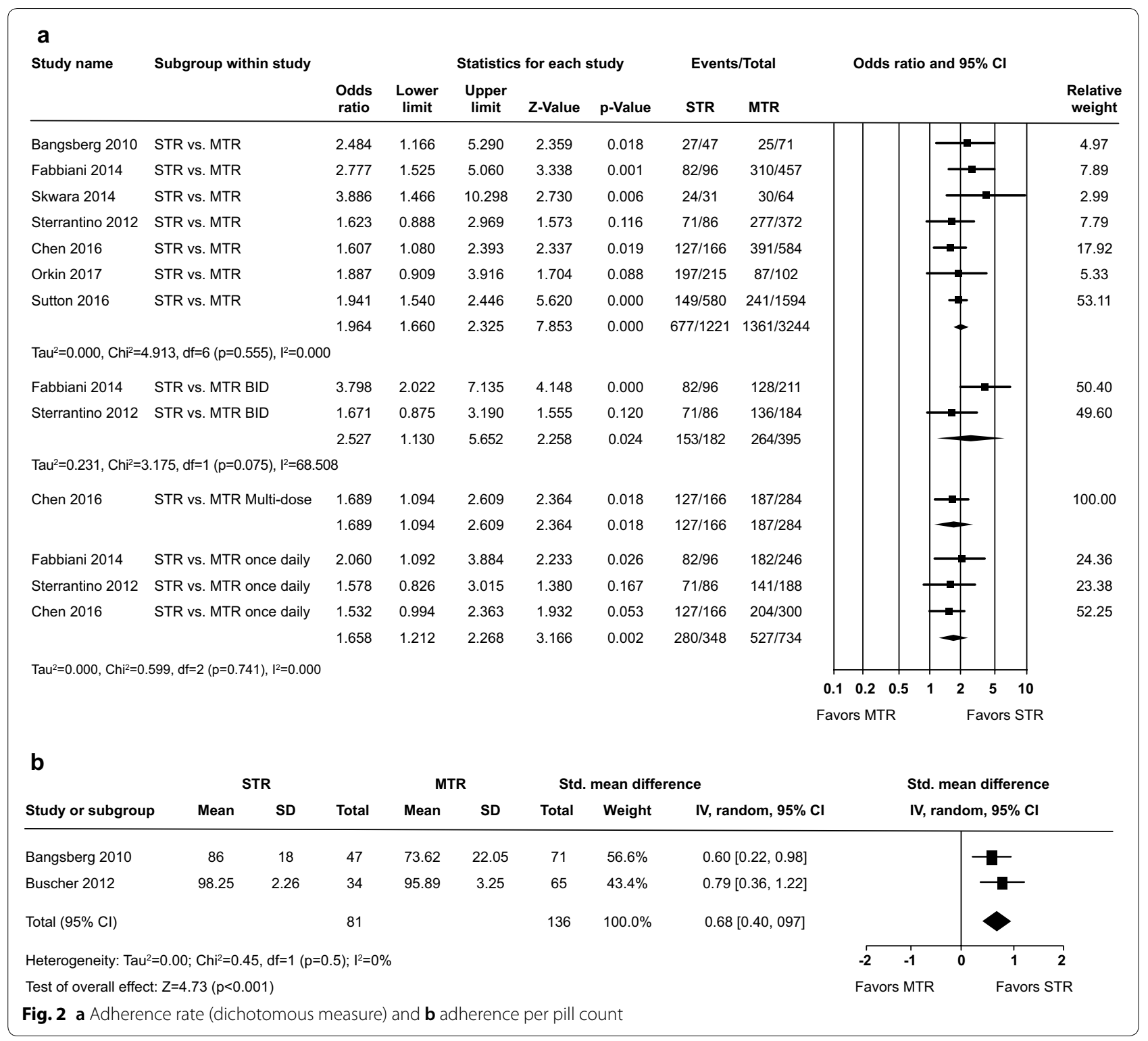

provided analyzable data for viral load suppression (defined per protocol $<50$ copies $/ \mathrm{ml}$ ) at 48 weeks and four studies $[25-27,30,31]$ reported change in CD4 cell count at 48 weeks. There was a statistically significant difference in viral load suppression at 48 weeks between the STR and MTR groups (relative risk (RR): 1.05 , 95\% CI $1.02-1.09, \mathrm{p}=0.002$ ) and low heterogeneity between the studies was observed $\left(\mathrm{Chi}^{2}=7.54\right.$; $\mathrm{i}^{2}=33.7 \%$ ) (Fig. 3a). The difference (SMD) in CD4 cell count between STR and MTR was not statistically significant at 48 weeks (SMD: 0.029, 95\% CI: $-0.06-0.12$, $\mathrm{p}=0.51$ ), and no heterogeneity between the studies was observed $\left(\mathrm{Chi}^{2}=1.76 ; \mathrm{i}^{2}=0.0 \%\right.$, Fig. $\left.3 \mathrm{~b}\right)$.

\section{Safety and tolerability outcomes}

Of the 63 studies, 37 reported safety outcomes with data relevant to adverse events (AE), laboratory abnormalities, mortality, and tolerability (treatment discontinuation). Six studies [22, 25-28, 32] reported analyzable data for the safety and tolerability outcome parameters. All six studies [22, 25-28, 32] reported rates of discontinuation due to any reason, three reported protocol-defined SAE $[22,25,26]$, five reported Grade 3 to $4 \mathrm{AE}[22,25,26,28$, 32], two reported Grade 3 to 4 laboratory abnormalities $[25,32]$ and two reported mortality $[25,26]$.

Meta-analyses of SAE, grade 3 to $4 \mathrm{AE}$ and mortality revealed no statistically significant differences between STR and MTR groups (Fig. 4a) with RR of any SAE (RR: 


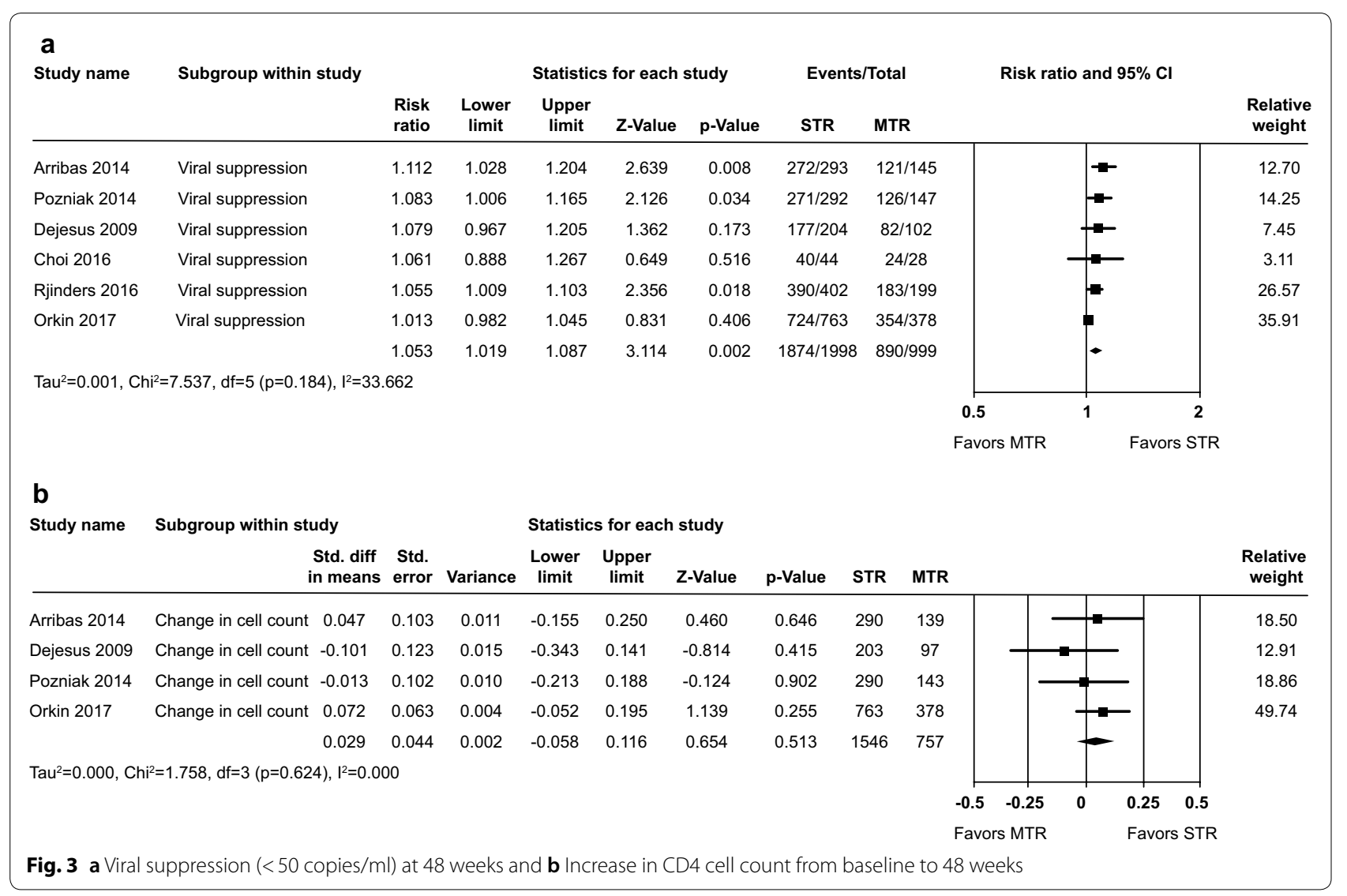

0.96, 95\% CI 0.64-1.45, $\mathrm{p}=0.86)$, Grade 3 to 4 AE (RR: $0.83,95 \%$ CI $0.59-1.17, \mathrm{p}=0.29$ ) and mortality (RR: 0.49, 95\% CI $0.05-4.65, \mathrm{p}=0.53)$ and minimal heterogeneity among the studies $\left(\mathrm{Chi}^{2}=0.17-4.55 ; \mathrm{i}^{2}=0.00-12.17 \%\right)$. Grade 3 to 4 laboratory abnormalities were significantly less likely for the STR group versus the collective MTR groups, RR: $0.68,95 \%$ CI $0.49-0.94, \mathrm{p}=0.02$, with no heterogeneity in the studies.

The risk of discontinuation due to any reason was lower in the STR group versus the MTR group, RR: 0.69, 95\% CI $0.47-1.00, \mathrm{p}=0.05,-$. 4B. Moderate heterogeneity was observed in the tolerability studies $\left(\mathrm{Chi}^{2}=9.71\right.$, $\left.\mathrm{i}^{2}=48.49 \%\right)$, potentially due to variation in study design and/or population.

\section{Humanistic or patient-reported outcomes (PRO)}

Seven [19, 20, 25, 27, 33-35] of the 63 studies reported evaluable results of PRO associated with ART. The most commonly used instruments in the studies included the HIV Treatment Satisfaction Score (HIV-TSQ), HIV Symptom Index (HIV-SI) and the 36-Item Short Form Health Survey (SF-36). Variability among the instruments used meant that the meta-analysis could not include PRO data. Five studies [25, 27, 33-35] reported positive impact with results favoring STR in patient satisfaction, symptom control and overall health status. Among these five studies, the impact of STR or MTR on mental health was rated as neutral. Among the five, only one directly compared STR and MTR [25]. In this study, STR was associated with a higher HIV-TSQ score as compared to MTR at weeks 4 ( 21.5 versus $13.3, \mathrm{p}<0.0001)$ through week 24 (23.1 versus 14.5, p<0.0001) [25]. Further, STR resulted in lower rates of patient-reported $\mathrm{AE}$ such as diarrhea (30\% versus 46\%, $\mathrm{p}<0.001)$ and bloating $(33 \%$ versus $41 \%, \mathrm{p}=0.039$ ) compared with $\mathrm{MTR}$ ( $\mathrm{p}<0.05$ for both) [25]. Similar improvements in the STR and MTR arms in the other two studies, regardless of pill burden $[19,20]$.

\section{Economic summary}

Thirteen [23, 36-47] of 63 studies reported economic outcomes. Ten studies were summarized in the previous review [11]. Three additional studies were identified and are reported in this publication [23, 44, 45]. One study, evaluating overall ART changes in $3850 \mathrm{PLWH}$, found that modifying therapy resulted in a mean additional cost of $€ 14$ (SD $€ 216$; range $-€ 528$ to $+€ 831$ ) per month per patient [44]. Toxicity and therapy simplifications were cited as the leading causes for regimen changes [44]. Economic outcomes have been modeled using simulations 


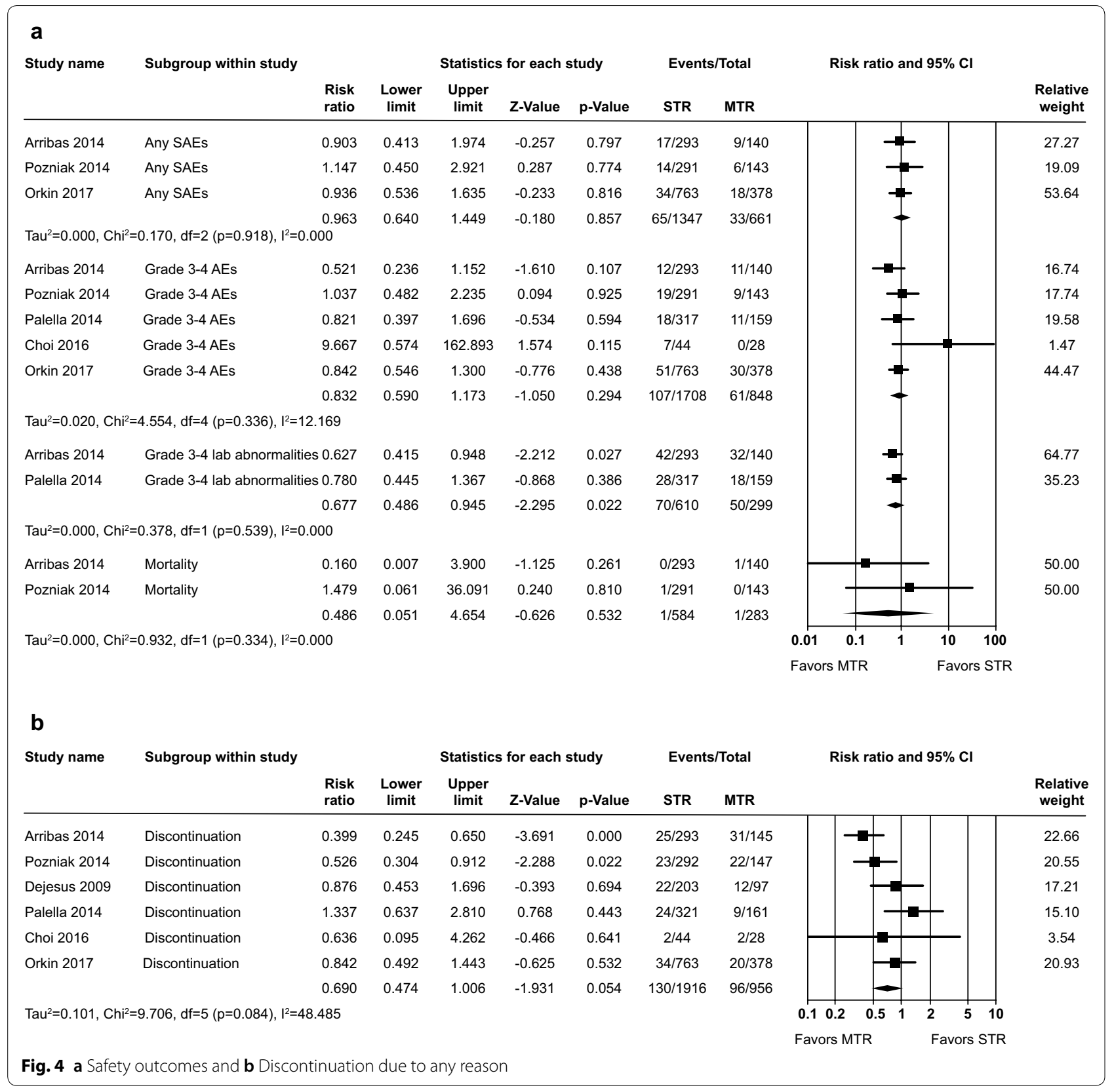

and insurance claims data including comprehensive computer-based microsimulation to compare the costeffectiveness of STR to MTR for initial treatment and concluded that the ICER of STR to MTR is $\$ 26,383$ per quality-adjusted life year [45]. A multivariate regression model study using Medicaid medical and pharmacy claims data was completed to determine the impact of ART pill burden in 2174 PLWH [23]. Patients taking STR had a lower risk of hospitalization (HR: 0.71, 95\% CI $0.59-0.86, \mathrm{p}=\mathrm{NS}$ ) and extended time to hospitalization (median: 1508 versus 1032 days, $\mathrm{p}=0.0042$ ) [23].

\section{Discussion}

The results with respect to adherence, viral load, Grade 3 to 4 laboratory abnormalities favor STR versus MTR. However, in contrast to previous review, the risk of discontinuation due to any reason was lower in the STR group, implying a better tolerability profile. Improved adherence and low discontinuation rates have been seen in other conditions when treatments are simplified that could be attributed to lower pill burdens and dosing frequencies [7]. Although the study was not designed to address the issue, it may be that patient satisfaction 
with STR may contribute to better adherence and lower rates of discontinuation, leading to improved clinical outcomes. This aspect, rarely reported in clinical trials, should be given enhanced consideration for inclusion.

Improved clinical outcomes could in turn result in lower resource utilization and cost effectiveness of therapy. In fact, the economic studies included in this update indicated lower risk of hospitalization, as well as an ICER of approximately $\$ 26,000$, which is within the range of willingness to pay thresholds [45]. It should be noted that these economic findings are based on OS and modelling, with inherent selection bias based on variations in patient characteristics and multiple assumptions which may or may not accurately reflect the treatment paradigm in a real-world setting.

We also examined humanistic outcomes based on patient reported data (not included in the meta-analysis), which was not carried out in the previous review. The findings were by and large positive for STR with respect to better patient satisfaction, symptom control and health status, including a lower rate of diarrhea and bloating. Given the weight that humanistic outcomes are gaining with third party payers and government funding bodies, it is worth considering when deciding regimen options. Further, consistent use of assessment tool or inclusion of multiple tools to permit higher order analyses is encouraged.

Similar to the previous review, the strengths of this update are based on the implementation of a welldefined search strategy and the use of a robust randomeffects model to assess pooled estimates extracted from both RCTs and observational studies. We only included studies which had quantifiable outcomes that could be included in an analysis. For this reason, we only included open label studies with 48-week outcomes data for the meta-analysis, to enable us to compare STR and MTR. We excluded double blinded, randomized studies where patients on STR would also receive placebo pills (to equal the total number of pills for patients on MTR), since this would not result in an accurate comparison. Authors acknowledge that different classes of medications were compared and factors such as the mechanism of action might also play a role in safety and efficacy results.

\section{Conclusion}

Patients on STR have better adherence, lower rates of discontinuation, improved viral load and fewer laboratory abnormalities than those on MTR. Economic and humanistic outcomes favor STR. Additional studies to examine the link between patient satisfaction, adherence and clinical outcomes in PLWH receiving STR or MTR, would provide additional evidence to support STR over MTR. In the meantime, it is important to implement and follow current global and country specific guidelines that specify STR as one of the primary recommended treatments in the management of PLWH. Improving access to STRs for patients, physicians and healthcare systems is critical, in improving the quality of life of PLWH.

\section{Additional files}

Additional file 1. Characteristics of studies included in qualitative evidence synthesis.

Additional file 2. Characteristics of study population included in the meta-analysis.

Additional file 3. a. Quality of RCTs Included in quantitative evidence synthesis (studies included in meta-analysis). b. Quality of observational studies included in quantitative evidence synthesis (studies included in meta-analysis).

\section{Authors' contributions}

All authors (PGC, WCY, CHM, ID, KLT, SS, DS) each substantially contributed to the conception or design of the work; the acquisition, analysis, or interpretation of the data for the work; drafting the work or revising it critically for important intellectual content; final approval of the version to be published; and agreement to be accountable for all aspects of the work in ensuring that questions related to the accuracy or integrity of any part of the work are appropriately investigated and resolved. All authors read and approved the final manuscript.

\section{Author details}

${ }^{1}$ University of North Texas System College of Pharmacy, 3500 Camp Bowie Blvd, Fort Worth, TX 76107, USA. ${ }^{2}$ Jannsen-Cilag GmbH, Johnson \& Johnson Platz 1, 41470 Neuss, Germany. ${ }^{3}$ Janssen EMEA, Turnhoutseweg 30, 2340 Beerse, Belgium. ${ }^{4}$ Janssen-Cilag Polska Sp. Z o.o, Iłżecka 24, 02-135 Warsaw, Poland. ${ }^{5}$ Market Access Solutions, LLC, 575 NJ-28, Raritan, NJ 08869, USA.

\section{Acknowledgements}

We would like to thank Melissa Stauffer, PhD for her support in developing and running the meta-analysis model.

\section{Competing interests}

PGC receives grant funding from Centers for Disease Control and Prevention and National Institute on Minority Health and Health Disparities to conduct practice-based research; is contracted on behalf of University of North Texas Health Science Center by Merck to conduct a Phase III clinical trial; serves on an advisory board or the speaker's bureau for GlaxoSmithKline, NAPO Pharmaceuticals and Merck \& Company, Inc; and has been compensated for consultations provided to National Institutes of Health, Agency for Healthcare Research and Quality, Center for Medicare and Medicaid Services, Prime Education, SIGA Consulting, 3D Communications, Market Access Solutions, and Ipsos. CHM, ID, and KLT are employed by Janssen. WCY, SS, and DS have no conflicts of interest.

\section{Availability of data and materials}

The datasets used and/or analyzed during the current study are available from the corresponding author on reasonable request.

\section{Consent for publication}

Not applicable.

Ethics approval and consent to participate

Not applicable.

\section{Funding}

This study was financially supported by Janssen EMEA. 


\section{Publisher's Note}

Springer Nature remains neutral with regard to jurisdictional claims in published maps and institutional affiliations.

Received: 3 August 2018 Accepted: 10 October 2018

Published online: 30 October 2018

\section{References}

1. AVERT. Global HIV and AIDS statistics. 2017. https://www.avert.org/globa I-hiv-and-aids-statistics.

2. U.S. Department of Health and Human Services. FDA approval of HIV medicines; 2016.

3. U.S. Department of Health and Human Services. Guidelines for the use of antiretroviral agents in HIV-1-infected adults and adolescents; 2016.

4. Centers for Disease Control and Prevention. Evolution of HIV/AIDS prevention programs-United States, 1981-2006. MMWR Morb Mortal Wkly Rep. 2006:55(21):597-603.

5. Sepkowitz KA. AIDS-the first 20 years. N Engl J Med. 2001;344:1764-72.

6. World Health Organization. Consolidated guidelines on the use of antiretroviral drugs for treating and preventing HIV infection. 2nd ed. Geneva: World Health Organization; 2016.

7. World Health Organization. Adherence to long-term therapies: evidence for action. Geneva: World Health Organization; 2003.

8. Deeks SG, Lewin SR, Havlir DV. The end of AIDS: hIV infection as a chronic disease. Lancet. 2013;382(9903):1525-33.

9. UNAIDS. Treatment 2015; 2015.

10. U.S. Department of Health and Human Services. HIV/AIDS glossary: boosting; 2017.

11. Clay PG, Nag S, Graham CM, Narayanan S. Meta-analysis of studies comparing single and multi-tablet fixed dose combination HIV treatment regimens. Medicine (Baltimore). 2015;94(42):e1677.

12. Moher D, Shamseer $L$, Clarke M, Ghersi D, Liberati A, Petticrew M, et al. Preferred reporting items for systematic review and meta-analysis protocols (PRISMA-P) 2015 statement. Syst Rev. 2015:4:1.

13. Higgins JPT GS, eds. Cochrane Handbook for Systematic Reviews of Interventions Version 5.0.2 (updated September 2009). The Cochrane Collaboration 2008

14. Critical Appraisal Skills Programme (CASP). 12 questions to help you make sense of cohort study. 2017.

15. DerSimonian R, Laird N. Meta-analysis in clinical trials. Control Clin Trials. 1986;7(3):177-88.

16. Higgins JP, Thompson SG. Quantifying heterogeneity in a meta-analysis. Stat Med. 2002;21(11):1539-58.

17. Bangsberg DR, Ragland K, Monk A, Deeks SG. A single tablet regimen is associated with higher adherence and viral suppression than multiple tablet regimens in HIV + homeless and marginally housed people. Aids. 2010;24(18):2835-40.

18. Fabbiani M, Zaccarelli M, Grima P, Prosperi M, Fanti I, Colafigli M, et al. Single tablet regimens are associated with reduced Efavirenz withdrawal in antiretroviral therapy naive or switching for simplification HIV-infected patients. BMC Infect Dis. 2014;14:26.

19. Skwara P, Bociaga-Jasik M, Kalinowska-Nowak A. Adherence to singletablet versus multiple-tablet regimens in the treatment of HIV infection: a questionnaire-based survey on patients satisfaction. HIV AIDS Rev. 2014;13:95-9.

20. Sterrantino G, Santoro L, Bartolozzi D, Trotta M, Zaccarelli M. Self-reported adherence supports patient preference for the single tablet regimen (STR) in the current CART era. Patient Prefer Adherence. 2012;6:427-33.

21. Chen Y, Chen K, Kalichman SC. Barriers to HIV medication adherence as a function of regimen simplification. Ann Behav Med. 2017;51(1):67-78.

22. Orkin C, DeJesus E, Ramgopal M, Crofoot G, Ruane P, LaMarca A, et al. Switching from tenofovir disoproxil fumarate to tenofovir alafenamide coformulated with rilpivirine and emtricitabine in virally suppressed adults with HIV-1 infection: a randomised, double-blind, multicentre, phase 3b, non-inferiority study. Lancet HIV. 2017;4(5):e195-204.

23. Sutton SS, Hardin JW, Bramley TJ, D'Souza AO, Bennet CL. Single-versus multiple tablet HIV regimens: adherence and hospitalization risks. Am J Manag Care. 2016;22:242-8.
24. Buscher A, Hartman C, Kallen MA, Giordano TP. Impact of antiretroviral dosing frequency and pill burden on adherence among newly diagnosed, antiretroviral-naive HIV patients. Int J STD AIDS. 2012;23(5):351-5.

25. Arribas JR, Pialoux G, Gathe J, Di Perri G, Reynes J, Tebas P, et al. Simplification to coformulated elvitegravir, cobicistat, emtricitabine, and tenofovir versus continuation of ritonavir-boosted protease inhibitor with emtricitabine and tenofovir in adults with virologically suppressed HIV (STRATEGY-PI): 48 week results of a randomised, open-label, phase $3 b$, non-inferiority trial. Lancet Infect Dis. 2014;14(7):581-9.

26. Pozniak A, Markowitz M, Mills A, Stellbrink HJ, Antela A, Domingo P, et al. Switching to coformulated elvitegravir, cobicistat, emtricitabine, and tenofovir versus continuation of non-nucleoside reverse transcriptase inhibitor with emtricitabine and tenofovir in virologically suppressed adults with HIV (STRATEGY-NNRTI): 48 week results of a randomised, open-label, phase 3b non-inferiority trial. Lancet Infect Dis. 2014;14(7):590-9.

27. Dejesus E, Young B, Morales-Ramirez JO, Sloan L, Ward DJ, Flaherty JF, et al. Simplification of antiretroviral therapy to a single-tablet regimen consisting of efavirenz, emtricitabine, and tenofovir disoproxil fumarate versus unmodified antiretroviral therapy in virologically suppressed HIV1-infected patients. J Acquir Immune Defic Syndr. 2009;51(2):163-74.

28. Choi JY, Sungkanuparph S, Anekthananon T, Sax P, DeJesus E, Edelstein H, et al. Efficacy and safety of Elvitegravir/Cobicistat/Emtricitabine/Tenofovir Disoproxil Fumarate in Asian subjects with human immunodeficiency virus 1 infection: a sub-analysis of phase 3 clinical trials. Infect Chemother. 2016:48(3):219-24.

29. Rijnders B, Post F, Rieger A. Longer-term renal safety of tenofovir alafenamide vs tenofovir disoproxil fumarate. Conference on retroviruses and opportunistic infections, February 22-25, 2016, Boston 2016.

30. Arribas JR, Thompson M, Sax PE, Haas B, McDonald C, Wohl A, et al. A randomized, double-blind comparison of Tenofovir Alafenamide (TAF) vs. Tenofovir Disoproxil fumarate (TDF), each coformulated with Elvitegravir, Cobicistat, and Emtricitabine (E/C/F) for Initial HIV-1 Treatment: week 144 results. J Acquir Immune Defic Syndr 2017;75:211-8.

31. Koenig E, Orkin C, Clarke A, Podzamczer D, Clotet B, Callebaut C, et al. Improved safety and efficacy of TAF versus TDF single-tablet regimen in HIV-1 treatment-naïve women through week 48. J Int AIDS Soc. 2016;19(Suppl 1):21083.

32. Palella FJ Jr, Fisher M, Tebas P, Gazzard B, Ruane P, Van Lunzen J, et al. Simplification to rilpivirine/emtricitabine/tenofovir disoproxil fumarate from ritonavir-boosted protease inhibitor antiretroviral therapy in a randomized trial of HIV-1 RNA-suppressed participants. Aids. 2014:28(3):335-44.

33. Huhn GD, Tebas P, Gallant J, Wilkin T, Cheng A, Yan M, et al. A Randomized, Open-Label trial to evaluate switching to Elvitegravir/Cobicistat/Emtricitabine/Tenofovir Alafenamide Plus Darunavir in treatment-experienced HIV-1-infected adults. J Acquir Immune Defic Syndr. 2017;74(2):193-200.

34. Airoldi M, Zaccarelli M, Bisi L, Bini T, Antinori A, Mussini C, et al. One-pill once-a-day HAART: a simplification strategy that improves adherence and quality of life of HIV-infected subjects. Patient Prefer Adherence. 2010;4:115-25.

35. Hanna DB, Hessol NA, Golub ET, Cocohoba JM, Cohen MH, Levine AM, et al. Increase in single-tablet regimen use and associated improvements in adherence-related outcomes in HIV-infected women. J Acquir Immune Defic Syndr. 2014;65(5):587-96.

36. Beck EJ, Mandalia S, Sangha R, Youle M, Brettle R, Gompels M, et al. Lower healthcare costs associated with the use of a single-pill ARV regimen in the UK, 2004-2008. PLOS ONE. 2012;7(10):e47376.

37. Grimes RM, Shenouda TA. Using cost as a consideration for antiretroviral regimen selection: an example using average wholesale prices. AIDS Care. 2013;25(11):1380-4

38. Homar F, Lozano V, Martinez-Gomez J, Oyaguez I, Pareja A, Payeras A, et al Cost analysis of HIV treatment and drug-related adverse events when fixed-dose combinations of antiretrovirals (FDCs) were stopped, versus continuation with FDCs. Health Econ Rev. 2012;2(1):16.

39. Angeletti C, Pezzotti P, Antinori A, Mammone A, Navarra A, Orchi N, et al. Antiretroviral treatment based cost saving interventions may offset expenses for new patients and earlier treatment start. HIV Med. 2014:15:165-74.

40. Colombo GL, Castagna A, Di Matteo S, Galli L, Bruno G, Poli A, et al. Cost analysis of initial highly active antiretroviral therapy regimens for 
managing human immunodeficiency virus-infected patients according to clinical practice in a hospital setting. Ther Clin Risk Manag. 2014;10:9-15.

41. Colombo GL, Di Matteo S, Antinori A, Medaglia M, Murachelli S, Rizzardini G. Economic evaluation of initial antiretroviral therapy for HIV-infected patients: an update of Italian guidelines. Clinicoecon Outcomes Res. 2013;5:489-96

42. Colombo GL, Di Matteo S, Maggiolo F. Antiretroviral therapy in HIVinfected patients: a proposal to assess the economic value of the singletablet regimen. Clinicoecon Outcomes Res. 2013;5:59-68.

43. Walensky RP, Sax PE, Nakamura YM, Weinstein MC, Pei PP, Freedberg $\mathrm{KA}$, et al. Economic savings versus health losses: the cost-effectivenes of generic antiretroviral therapy in the United States. Ann Intern Med. 2013;158(2):84-92.
44. Sorbino-Jiménez C, Jiménez-Nácher I, Moreno-Ramos F. Analysis of antiretroviral therapy modification in routine clinical practice in the management of HIV infection. Eur J Hosp Pharm. 2017;24:96-100.

45. Sweet DE, Altice FL, Cohen CJ, Vandewalle B. Cost-effectiveness of singleversus generic multiple-tablet regimens for treatment of HIV-1 infection in the United States. PLoS ONE. 2016;11(1):e0147821.

46. Cohen CJ, Meyers JL, Davis KL. Association between daily antiretroviral pill burden and treatment adherence, hospitalisation risk, and other healthcare utilisation and costs in a US medicaid population with HIV. BMJ Open. 2013;3(8):e003028.

47. Juday T, Gupta S, Grimm K, Wagner S, Kim E. Factors associated with complete adherence to HIV combination antiretroviral therapy. HIV Clin Trials. 2011;12(2):71-8.
Ready to submit your research? Choose BMC and benefit from:

- fast, convenient online submission

- thorough peer review by experienced researchers in your field

- rapid publication on acceptance

- support for research data, including large and complex data types

- gold Open Access which fosters wider collaboration and increased citations

- maximum visibility for your research: over $100 \mathrm{M}$ website views per year

At BMC, research is always in progress.

Learn more biomedcentral.com/submissions 